CASO CLÍNICO

\title{
Carcinoma adrenal pediátrico: presentación de un caso y revisión de la literatura
}

\section{Pediatric adrenal carcinoma: case report and literature review}

Miguel A. Solís-Guadarrama1*, Dania G. Solís-Cano', Ana C. Amador-Martínez', Javier Baquera-Heredia ${ }^{2}$ y Guilermo Molinar-Flores ${ }^{2}$

${ }_{1}^{1}$ Imagenología Diagnóstica y Terapéutica; ${ }^{2}$ Patología. Centro Médico ABC, The American British Cowdray Medical Center, Ciudad de México, México

\section{RESUMEN}

Los carcinomas adrenales son raros, tienen una incidencia de 0.6-1.67 casos por cada millón de habitantes al año. Presentamos el caso de un paciente de 6 años de edad con malestar general y hepatomegalia. Durante su abordaje se solicitaron distintos estudios de imagen, entre ellos radiografía abdominal, ultrasonido hepático, resonancia magnética y tomografía por emisión de positrones-tomografía computarizada. Este caso permite revisar las manifestaciones radiológicas del carcinoma adrenal en pediatría mediante distintas técnicas. incluyendo la resonancia magnética y la medicina nuclear.

Palabras clave: Carcinoma. Adrenal. Pediátrico. Reporte de caso. 


\section{ABSTRACT}

Adrenal carcinomas are rare, they have an incidence of 0.6-1.67 cases per million habitants per year. We present the case of a 6-year-old patient with general malaise and hepatomegaly. During its approach, different imaging studies were requested, including abdominal radiography, liver ultrasound, magnetic resonance and positron emission tomography-computed tomography. This case allows us to see the radiological manifestations of adrenal carcinoma in pediatrics through different techniques, magnetic resonance and nuclear medicine.

Key words: Carcinoma. Adrenal. Pediatric. Case report.

\section{INTRODUCCIÓN}

Los carcinomas adrenales son raros, tienen una incidencia de 0.5 a 2 casos por cada millón de habitantes al año ${ }^{1}$, los tumores adrenocorticales representan solo el $0,2 \%$ de todos los cánceres malignos en niños y adolescentes ${ }^{2}$. La relación mujeres hombres es de 2.5-3-1. Se presenta en dos picos: en la primera década de vida y entre la cuarta y quinta décadas de la vida. Aproximadamente el $75 \%$ de los casos pediátricos se observan en los primeros 5 años $^{3}$.

\section{PRESENTACIÓN DEL CASO}

Paciente de sexo masculino, de 6 años de edad y sin antecedentes médicos de importancia, que inicia su padecimiento con fiebre intermitente acompañada de malestar general. Un médico facultativo inicia manejo viral con loratadina y fenilefrina por 3 días el cual remite; Los 3 meses siguientes persiste con fiebre recurrente que se trata con antibióticos, aciclovir y antipiréticos, la cual remitió; sin embargo, al cuarto mes presenta nuevamente fiebre de tipo intermitente de predominio nocturno, que se vuelve de difícil control y de alto grado, motivo por el cual se ingresa al servicio de urgencias, donde se interconsulta al infectólogo, y en la exploración física se detecta hepatomegalia.

Se solicitó ultrasonido abdominal (Figs. 1 y 2) en el que se identificó una lesión suprarrenal derecha de $13 \times 9 \mathrm{~cm}$, de aspecto sólido, con bordes parcialmente definidos y contenido heterogéneo a expensas de calcificaciones, así como con escasa vascularidad y mínima cantidad de líquido libre abdominal. Se decidió el ingreso hospitalario para la realización de una resonancia magnética (RM) (Figs. 3 y 4), en la que se corroboraron los mismos hallazgos, así como zonas con degeneración quística. Posteriormente, se hizo una tomografía por emisión de positrones-tomografía computarizada (PET-TC) con 68 Ga DOTANOC (Fig. 5), que identificó expresión heterogénea de receptores de somatostatina con aparente origen adrenal. Con los hallazgos se planeó laparotomía exploratoria y resección del tumor (Fig. 6). Tras la revisión patológica correspondiente se determinó el diagnóstico final de carcinoma adrenal.

\section{DISCUSIÓN}

Las neoplasias adrenocorticales pediátricas suelen aparecer antes de los 5 años de edad, 


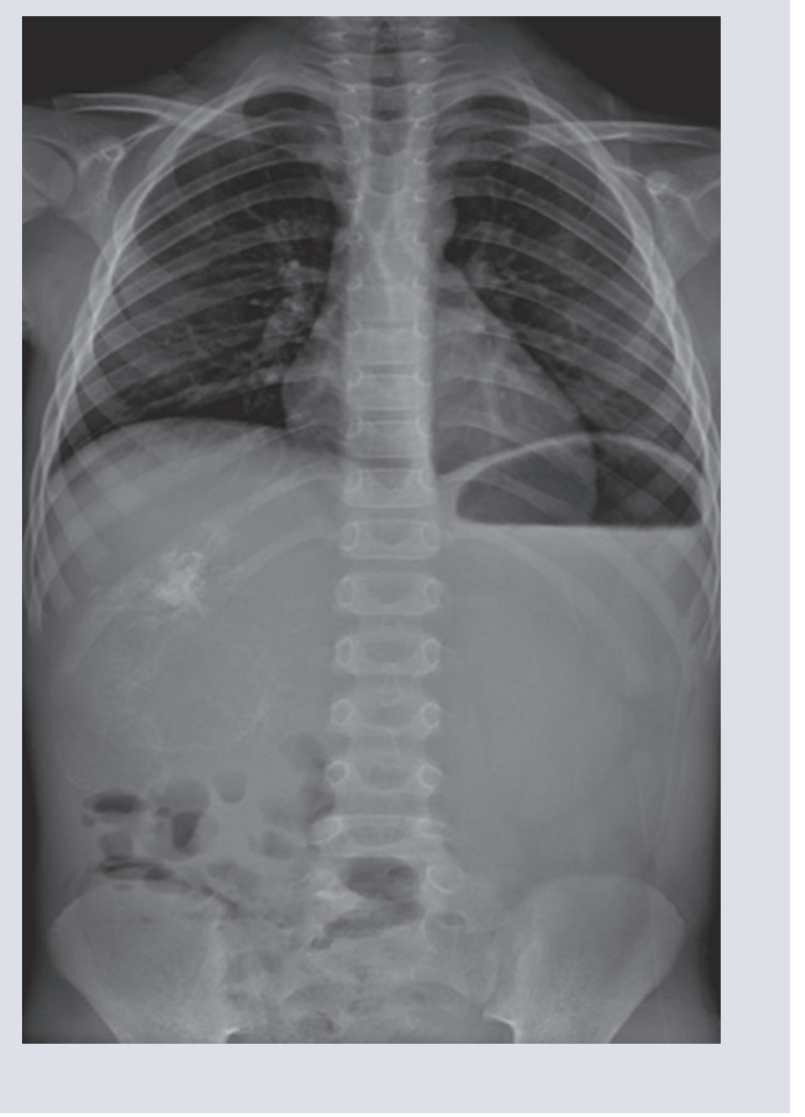

Figura 1. Radiografía toracoabdominal en proyección posteroanterior. Se identifican calcificaciones en el hipocondrio derecho.

afectan a las niñas con mayor frecuencia que a los niños ${ }^{4}$. Los carcinomas adrenales pueden asociarse a algunos síndromes como Li-Fraumeni, Beckwith-Wiedemann e hiperplasia adrenal congénita. El 50\% de los carcinomas adrenales son funcionales y se relacionan con el síndrome de Cushing y virilización ${ }^{3}$.

Las manifestaciones clínicas y el comportamiento biológico de estas lesiones pueden ser bastante diferentes de sus homólogos histológicamente similares en la población adulta, lo que hace que los criterios histopatológicos para distinguir los tumores benignos de los malignos sean equívocos ${ }^{5}$. Aunque la relación entre las características patológicas y el resultado clínico está bien establecida en las neoplasias adrenocorticales de adultos, el valor pronóstico de estas características en las neoplasias adrenocorticales pediátricas no está claro. El tipo histológico y el peso del tumor son los factores predictivos más confiables de resultados en neoplasias adrenocorticales pediátricas ${ }^{6}$.

La manifestación más común en la edad pediátrica es la virilización, que puede ocurrir sola o en combinación con el desequilibrio de hormonas suprarrenales ${ }^{2}$. Los carcinomas contabilizan hasta el 27\% de los casos de síndromes de Cushing ACTH-independientes (hormona adrenocorticotropa).

La invasión a la vena adrenal, vena cava, riñón adyacente y retroperitoneo es frecuente en el momento de presentación ${ }^{7}$. Los sitios más comunes de enfermedad metastásica son el hígado $(60 \%)$, ganglios linfáticos regionales (40\%), pulmones (40\%), pleura, huesos y piel $^{3}$.

En 2005, The European Network for the Study of Adrenal Tumors propuso una serie de procedimientos para los casos de sospecha de carcinoma adrenal. Entre los estudios de laboratorio se incluyen cortisol basal, ACTH, sulfato de dehidroepiandrostenediona, 17-hidroxiprogesterona, androstenediona, testosterona, estradiol, prueba de supresión con dexametasona y cortisol libre en orina. Los estudios de imagen recomendados para el diagnóstico incluyen TC o RM de abdomen y TC de tórax, escintigrafía en caso de metástasis óseas y tomografía por emisión de positrones con fluorodeoxiglucosa (PET FDG) como evaluación opcional. Para el seguimiento se indica la realización de TC o RM de abdomen y TC de tórax ${ }^{2}$. 

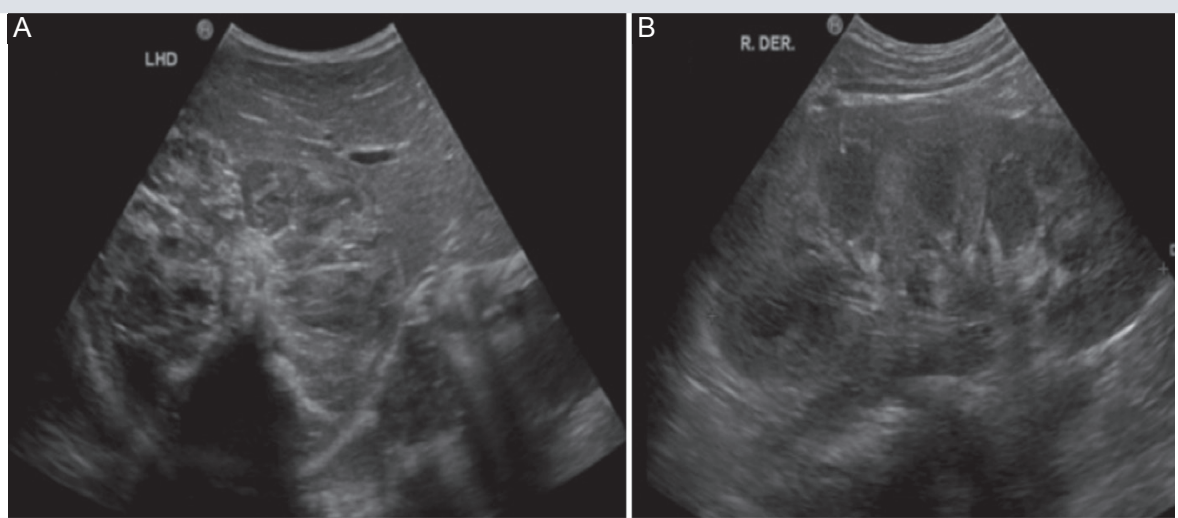

Figura 2. Ultrasonido en escala de grises con transductor convexo. A: Adyacente al lóbulo derecho, se observa una imagen redonda de bordes parcialmente definidos con aparente interfase con el parénquima hepático, de contenido heterogéneo a expensas de calcificaciones, aspecto sólido, medidas calculadas de 13 × $9 \mathrm{~cm}$. B: Riñón derecho en corte longitudinal, con eje mayor de 89 mm. Su contorno es regular, la relación corticomedular es adecuada, el sistema colector se observa sin dilatación.

El tamaño tumoral puede ser una herramienta útil para la diferenciación de las masas adrenales, la mayoría de los carcinomas adrenales tienen una media mayor a $11 \mathrm{~cm}$, mientras que los adenomas generalmente miden menos de $5 \mathrm{~cm}$. Los tumores de entre 3 y $10 \mathrm{~cm}$ pueden ser un reto diagnóstico.

Las imágenes son una importante herramienta para evaluar la resecabilidad y planificación quirúrgica ${ }^{8}$, la necesidad de una biopsia de tumor debe ser discutida críticamente $^{9}$ debido a que la escisión total es el factor pronóstico positivo más importante en estos $\operatorname{casos}^{8}$. Actualmente, ningún método de imagen puede caracterizar con certeza un carcinoma adrenal, sin embargo, la TC parece ser la herramienta de diagnóstico más útil ${ }^{10}$. Se ha establecido que una lesión adrenal es benigna cuando tiene de $10 \mathrm{o}$ menos UH (unidades Hounsfield) en fase simple y aquellas que poseen lavado de más del 50\% después de 15 minutos $^{11}$. La RM podría conferir hallazgos similares, no

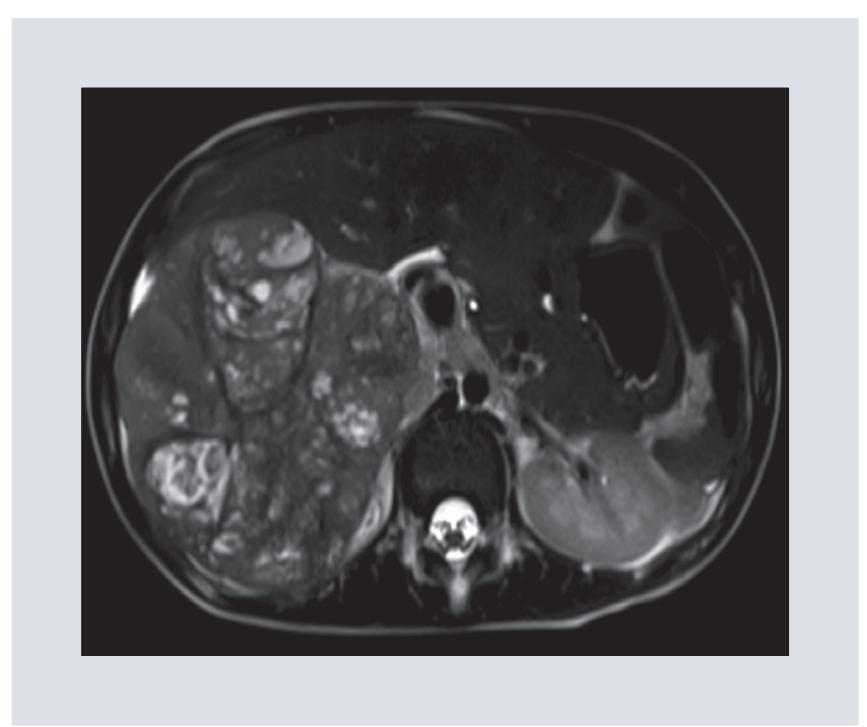

Figura 3. Resonancia magnética en corte axial ponderada en T2. Se identifica una lesión intraabdominal de bordes lobulados, de señal heterogénea, con áreas quísticas en su interior. Imágenes redondas en la región central que sugieren calcificaciones.

obstante, hay pocos resultados publicados al respecto $^{12}$.

Los hallazgos por TC incluyen una masa bien definida y con bordes invasivos, usualmente unilateral (bilateral en el 10\% de los casos), áreas de necrosis, hemorragia, calcificaciones 

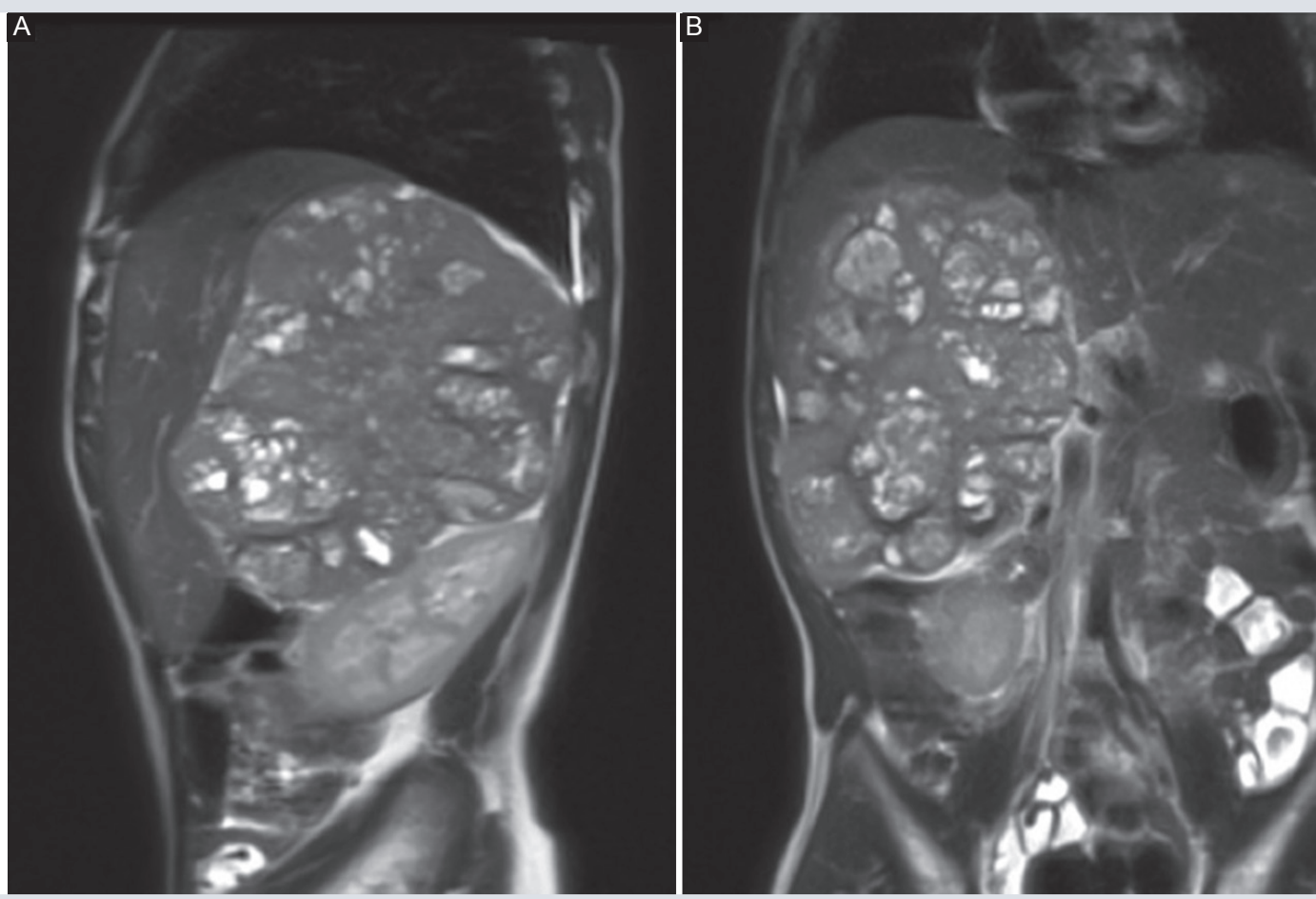

Figura 4. Resonancia magnética. Corte sagital ponderado en T2 (A), corte coronal ponderado en T2 (B). El hígado se encuentra desplazado en sentido anterior y superior, se observa una lesión en el segmento 2 de señal hiperintensa y con realce posterior a la administración de contraste en fase arterial y, posteriormente, es isointenso al resto del parénquima, dimensiones máximas de 12.2 × $9.5 \times 8.5$ en sus ejes longitudinal, anteroposterior y transverso, respectivamente.

(30\%), reforzamiento variable, invasión venosa (vena renal y vena cava inferior), metástasis al parénquima renal adyacente, metástasis a distancia (pulmones, hígado, ganglios linfáticos) y lavado similar a metástasis.

Por RM se observa como una masa adrenal hipointensa respecto al parénquima hepático en T1 e hiperintensa en T2. Tras la aplicación de contraste el reforamiento es variable debido a las zonas de necrosis ${ }^{13}$.

Elultrasonidonohasido plenamenteinvestigado, no obstante, podría usarse para la detección de metástasis hepáticas, así como para el seguimiento en pacientes seleccionados ${ }^{2}$. El uso de PET se reserva para aquellos casos de difícil diagnóstico, ya que virtualmente todos los carcinomas adrenales presentan captación de 18-FDG, a pesar de lo anterior, también es conocido que esto mismo sucede en la mayoría de las metástasis adrenales, feocromocitomas e, incluso, en algunos adenomas ${ }^{2}$.

Se ha propuesto el uso de análogos de somatostatina marcados como el 68Ga-DOTATOC y 68Ga-DOTATATE para el diagnóstico de tumores neuroendocrinos debido a la sobreexpresión de dichos receptores en este tipo de tumores ${ }^{14}$.

Acorde al mismo ENSATla, los estadios 1 y 2 corresponden a aquellas lesiones de $\leq 5 \mathrm{y}$ $>5 \mathrm{~cm}$, respectivamente. El estadio 3 se 

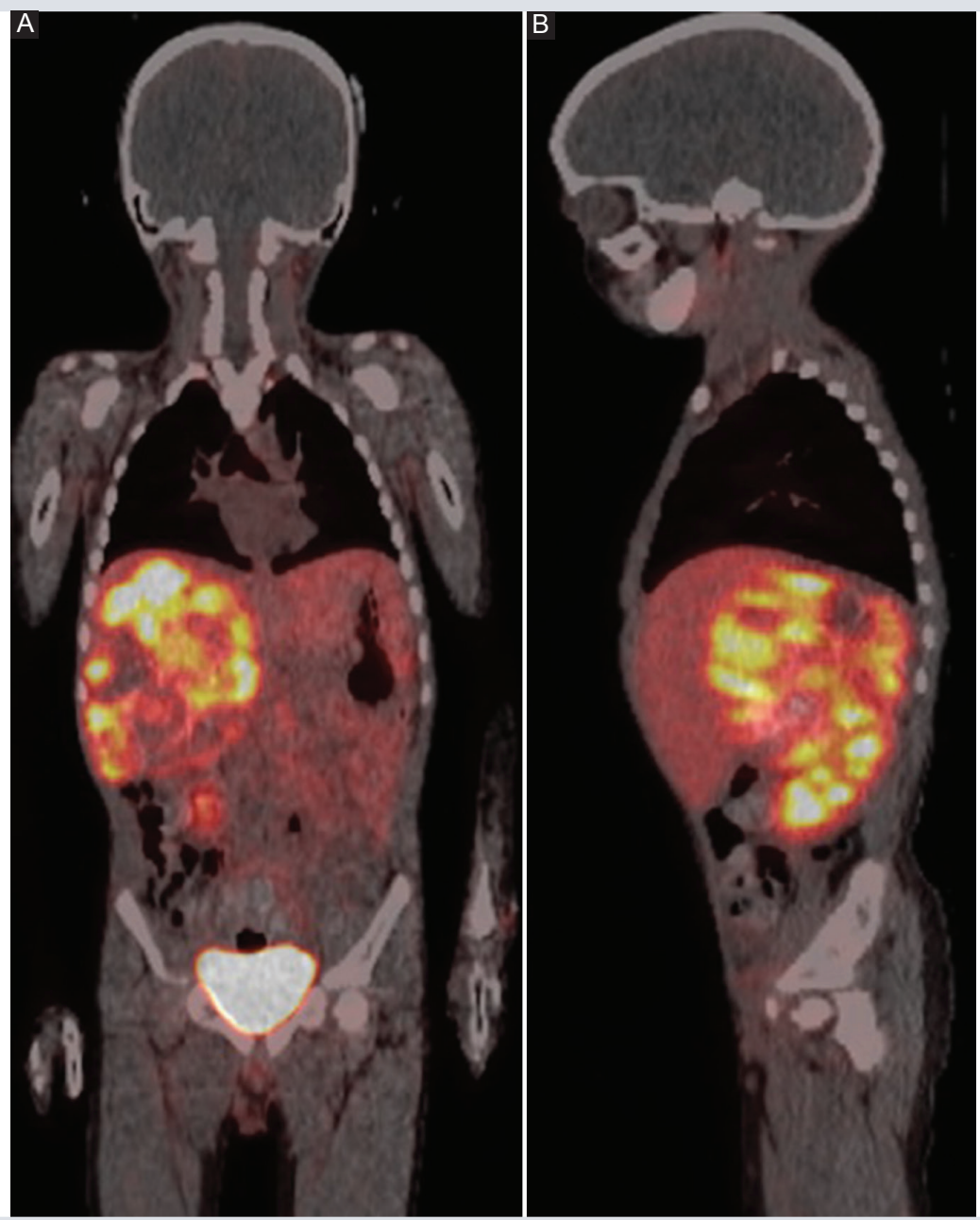

Figura 5. Tomografía por emisión de positrones-tomografía computarizada corporal con 68Ga-D0TANOC. Corte coronal (A), corte sagital (B). Lesión intraabdominal en el hipocondrio y flanco derecho, de bordes lobulados, heterogénea, que desplaza estructuras abdominales, sin identificar adecuado plano graso de separación con el lóbulo hepático derecho, polo superior del riñón, sin identificar la glándula adrenal ipsilateral, con dimensiones de $10 \times 11 \mathrm{~cm}$, que presenta zonas fotopénicas en su interior, en relación con necrosis, con SUV máximo de 14.8, expresa receptores de somatostatina de forma heterogénea.

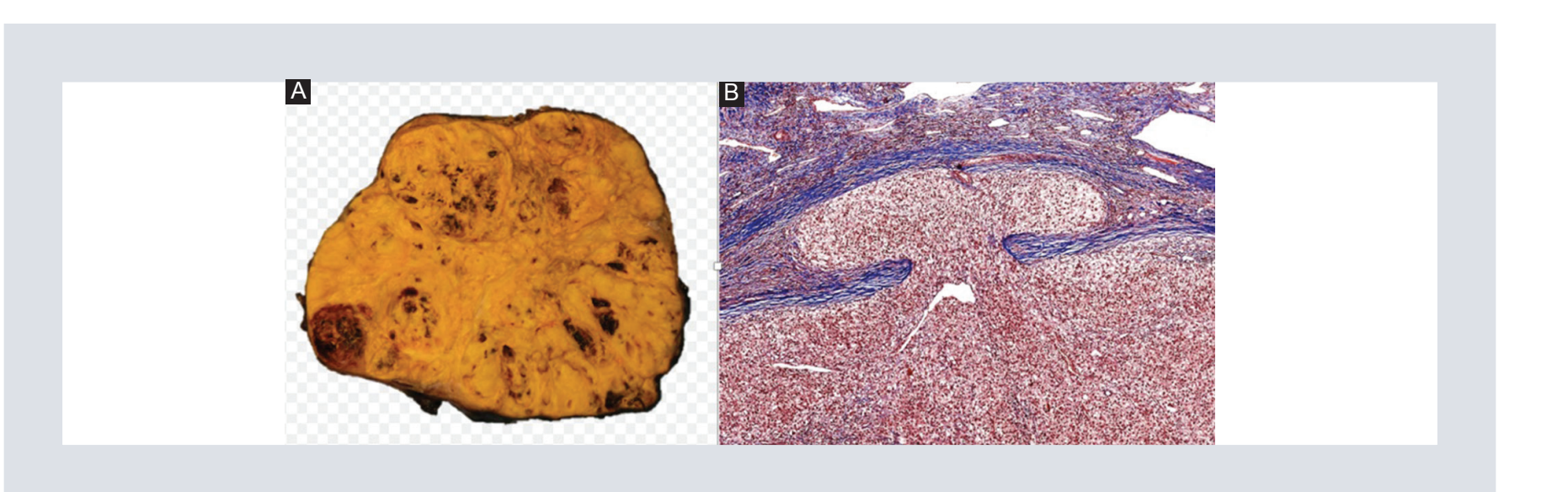

Figura 6. A: Espécimen de tumor retroperitoneal. B: Carcinoma adrenal cortical de bajo grado, con extensión extracapsular. 
caracteriza por invasión a tejidos adyacentes, adenopatías regionales y trombosis de las venas renal o cava inferior. El estadio 4 se restringe a aquellos pacientes con metástasis a distancia ${ }^{2}$.

\section{CONFLICTO DE INTERESES}

Los autores no reportan conflicto de interés.

\section{RESPONSABILIDADES ÉTICAS}

Protección de personas y animales. Los autores declaran que para esta investigación no se han realizado experimentos en seres humanos ni en animales.

Confidencialidad de los datos. Los autores declaran que en este artículo no aparecen datos de pacientes.

\section{Derecho a la privacidad y consentimiento} informado. Los autores han obtenido el consentimiento informado de los pacientes y/o sujetos referidos en el artículo. Este documento obra en poder del autor de correspondencia.

\section{BIBLIOGRAFÍA}

1. Permana H, Darmawan G, Ritonga E, Kusumawati M, Miftahurachman M, Soetedjo NN. An Interesting Case of Hepatic Adrenocortical Carcinoma. Acta Med Indones. 2018;50(3)257-9.

2. Fassnacht M, Kroiss M, Allolio B. Update in Adrenocortical Carcinoma. J Clin Endocrinol Metab. 2013;98(12):4551-64.

3. Adam A, Dixon A, Gillard J, Schaefer-Prokop C, Grainger R. Grainger \& Allison's Diagnostic Radiology. Chapter 4. Churchill Livingstone; 2015, pp.1008-33.

4. Agrons GA, Lonergan GJ, Dickey GE, Perez-Monte JE. Adrenocortical neoplasms in children: radiologic-pathologic correlation. Radiographics. 1999;19:989-1008.

5. Wieneke JA, Thompson LD, Heffess CS. Adrenal cortical neoplasms in the pediatric population: a clinicopathologic and immunophenotypic analysis of 83 patients. Am J Surg Pathol. 2003;27:867-881.

6. Bugg MF, Ribeiro RC, Roberson PK Lloyd RV, Sandrini R, Silva JB. Correlation of pathologic features with clinical outcome in pediatric adrenocortical neoplasia. A study of a Brazilian population. Brazilian Group for Treatment of Childhood Adrenocortical Tumors. Am J Clin Pathol. 1994;101:625-9

7. Godine LB, Berdon WE, Brasch RC, Leonidas JC. Adrenocortical carcinoma with extension into inferior vena cava and right atrium: report of 3 cases in children. Pediatr Radiol. 1990;20:166-8; discussion 169.

8. Ribeiro J, Ribeiro RC, Fletcher BD. Imaging findings in pediatric adrenocortical carcinoma. Pediatr Radiol. 2000;30:45-51.

9. Hubertus J, Boxberger N, Redlich A, von Schweinitz D, Vorwerk P, et al. Surgical aspects in the treatment of adrenocortical carcinomas in children: data of the GPOH-MET 97 trial. Klin Padiatr. 2012;224:143-7.

10. Neblett WW, Frexes-Steed M, Scott HW Jr. Experience with adrenocortical neoplasms in childhood. Am Surg. 1987;53:117-25.

11. Caoili EM, Korobkin M, Francis IR, Cohan RH, Platt JF, Dunnick NR, et al. Adrenal masses: characterization with combined unenhanced and delayed enhanced CT. Radiology. 2002;222:629-33.

12. Hanson JA, Weber A, Reznek RH, Cotterill AM, Ross RJ, Harris RJ, et al. Magnetic resonance imaging of adrenocortical adenomas in childhood: correlation with computed tomography and ultrasound. Pediatr Radiol. 1996;26:794-9.

13. Bharwani N, Rockall A, Sahdev A, Gueorguiev M, Drake W, Grossman AB, et al. Adrenocortical Carcinoma: The Range of Appearances on CT and MRI. AJR Am J Roentgenol. 2011;196(6):W706-14.

14. Poeppel TD, Binse I, Petersenn S, Lahner H, Schott M, Antoch G, et al. 68Ga-DOTATOC Versus 68Ga-DOTATATE PET/CT in Functional Imaging of Neuroendocrine Tumors. J Nucl Med. 2011;52:1864-70. 